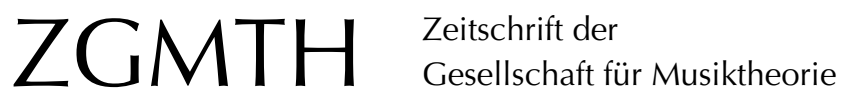

Froebe, Folker (2007): Historisches Panoptikum der Satzmodelle. ZGMTH 4/1-2, 185195. https://doi.org/10.31751/253

(C) 2007 Folker Froebe

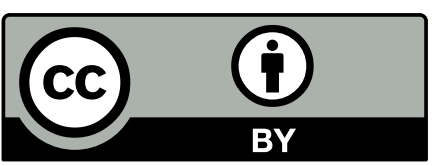

Dieser Text erscheint im Open Access und ist lizenziert unter einer Creative Commons Namensnennung 4.0 International Lizenz.

This is an open access article licensed under a

Creative Commons Attribution 4.0 International License.

veröffentlicht / first published: 01/01/2007

zuletzt geändert / last updated: 03/08/2009 


\title{
KOLUMNE
}

\section{Historisches Panoptikum der Satzmodelle}

\author{
Folker Froebe
}

Als musikalisch 'Spätberufener` kompensierte ich das im Kindesalter Versäumte durch Neugier und autodidaktischen Lernwillen: Mit 15 oder 16 Jahren dürfte ich so ziemlich alles verschlungen haben, was mir seinerzeit (in den 80er Jahren des 20. Jahrhunderts) an Theorielehrbüchern erreichbar war. Dieser frühen Lektüre verdanke ich viel, doch bei meinen Versuchen im Improvisieren, Komponieren und Generalbassspielen ließen Maler, Abraham, de la Motte, Ratz und all die anderen mich ziemlich allein. Als Student in Quellentexte eingewiesen zu werden, wurde für mich zu einem Schlüsselerlebnis: Die Schriften von Berardi, Werckmeister, Marpurg und Albrechtsberger spiegelten meine Erfahrungen und gaben jenen Satzmodellen, die bis dahin - namenlos und ungeordnet - mehr meinen Fingern als meinem Kopf vertraut waren, eine innere Ordnung und eine Heimat in meinem Bücherregal. Seither erkläre ich mich mit jeder Quellenlektüre von Neuem zum Zeitgenossen auf Probe.

Würde die Kluft von Jahrhunderten einfach übergangen, so bliebe sie unüberwindbar: Wieviele Leerstellen in den Texten verdanken sich den Konventionen gelehrten und lehrenden Schreibens oder der stillschweigenden Voraussetzung von seinerzeit Selbstverständlichem? Wieviel bloß Angedeutetes bedurfte auch vor 300 Jahren schon der Entfaltung in mündlich-praktischen Vermittlungszusammenhängen? Wer schlicht fragt, swas in den Quellen stehtı, kann - gerade auf der Suche nach Satzmodellen - Enttäuschungen erleben: Modellhaftes wird in disparaten Disziplinen und Lehrkontexten thematisiert,

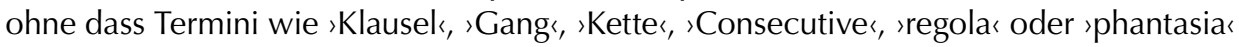
in einen konsistenten Modellbegriff mündeten. Und obgleich Modelle in Exempeln und »Ton-Mustern« (Spiess) allgegenwärtig sind, werden sie kaum je als solche angesprochen: Fast immer bleibt die wortsprachliche Darstellung hinter jenem Mehrwert zurück, den erst die Korrelation von Wort und Exempel (textimmanent) bzw. Theorie- und Kompositionsgeschichte (textübergreifend) offenbart. Wer Dokumente historischer Musiktheorie angemessen interpretieren will, muss jenes musikalische Denken zu neuem Leben erwecken, das in den Texten selbst nur noch mittelbar greifbar ist.

Die Rekonstruktion, Vergegenwärtigung und Fortschreibung der historischen Musiktheorie sind zentrale Aufgaben einer Musiktheorie, die den Status einer `Theorie - und dazu gehört der systematische Anspruch ebenso wie die (wissenschafts-)geschichtliche Reflexion - mit Recht für sich in Anspruch nehmen möchte. Vor diesem Hintergrund meine erste Leseempfehlung: »Music Theory and its Histories« (1993), ein mittlerweile sklassischer Artikel, in dem Thomas Christensen die Dahlhaus'sche Dichotomie von 
'historischem Interpretationsmuster und snormativer Theorie in einer hermeneutischen, auf `Horizontverschmelzung`(Gadamer) gerichteten Lektüre historischer Theorietexte aufgehoben wissen möchte.

Bei welchen Autoren lassen sich besonders gut „Clausulen abstehlen und ausspicken « ${ }^{1}$ ? Wer nach den Wurzeln des smodellgestützten diminutiven Kontrapunkts sucht, wird zuallererst fündig bei den Italienern des 16 . und 17. Jahrhunderts. ${ }^{2}$ Darüber hinaus konsultiere ich mit Vorliebe eine Reihe >deutscher Quellen, deren (wenigstens in der ersten Hälfte des 18. Jahrhunderts unverkennbarer) Konservativismus auch als Vorzug gelten mag, lassen sie doch in dem Bemühen, stilistische Veränderungen an das überkommene kontrapunktische Paradigma zu binden, zugleich eine entwicklungsgeschichtliche Kontinuität erkennen. Hier eine Auswahl meiner liebsten Kompositionslehren zwischen 1600 und 1800:

- Jan Pieterszoon Sweelinck, Composition-Lehre3: Zarlino im Konzentrat, ımodernisiertı und um zahlreiche selbständige Exempel bereichert.

- Johann Andreas Herbst, Musica Poetica, Sive Compendium Melopoëticum (Nürnberg 1643): "eine ganze Kompositionslehre auf Grundlage der Clausula formalis« (Braun). Knappgefasst, sehr klar und nah am Gegenstand, vor allem aber voller schöner Klauseln, Imitationsmodelle und `Gänge`.

- Andreas Werckmeister, Harmonologia musica (Frankfurt und Leipzig 1702): Hier schreibt einer mit dem Sendungsbewusstsein des gelehrten Praktikers, der frei von den Zwängen der Tradition zeigen möchte, 'wie's wirklich geht: : Wer aber dem fundament selber nachgehet, und weiter nachsuchen will, der wird sich an die Weitläufigkeit der Regeln nicht binden lassen [...].» ${ }^{4}$ Werckmeisters ^Highlightı: eine modellgestützte Kanon- und Fugenlehre in der Nachfolge des `Contrapunto alla mente.$^{5}$

- Mauritius Vogt, Conclave thesauri magnae artis musicae (Prag 1719): Vogts »Phantasia simplex« kommt einer >modernen Passagen (Sequenzen), so Vogt, lassen sich aus einer Phantasia sowohl »deduzieren« als auch auf eine solche $»$ reduzieren ${ }^{6}{ }^{6}$

- Franz Xaver Murschhauser, Academia musico-poetica bipartita (Nürnberg 1721): weder große Theorie noch herausragende Didaktik, dafür aber interessante Ausführungen zur Dissonanzenbehandlung und eine der reichsten Klauselsammlungen des 18. Jahrhunderts - noch Marpurg und Riepel zitieren Murschhausers sequenziell sausgeflohene Klauseln.

1 Herbst 1643, 115.

2 Vgl. Froebe 2007.

3 Sweelinck 1901.

4 Werckmeister 1702, Vorrede, 2.

5 Ebd., 95 ff., "Zugabe oder Anhang vom gedoppelten Contrapunct und fugis ligatis«.

6 Vogt 1719, $154 \mathrm{ff}$ - Eine kommentierte Teilübersetzung wurde nach dem Erscheinen dieser Kolumne vorgelegt (Froebe 2008). 
- Meinrad Spiess, Tractatus musicus compositorio-practicus (Augsburg 1745): eine unterschätzte Kompositionslehre, die sich vor den ıSpäteren` nicht verstecken muss. So ziemlich jeder Gegenstand der Lehre wird von Spiess genutzt, um - gewissermaßen nebenbei - Satzmodelle zu exemplifizieren.

- Friedrich Wilhelm Marpurg, Abhandlung von der Fuge (Berlin 1753/54) und Handbuch bey dem Generalbasse und der Composition (Berlin 1755-60): modellgestützte sars combinatoria vom Feinsten - noch Schumann hat seinen Kontrapunkt von Marpurg gelernt.

- Joseph Riepel, Anfangsgründe zur musicalischen Setzkunst (Regensburg/Wien 175286): Eine so bruchlose Einheit von handwerklicher Instruktion und ästhetischem Diskurs war wohl nur in diesem einen historischen Augenblick - an der Schwelle zwischen barocker Rhetorik und aufgeklärt rezeptionsorientierter Ästhetik - möglich. Riepels unausgesprochenes Credo: „Benutze Modelle und verbirg sie! «7 Für die gegenwärtige Lehre eine Quelle von unschätzbarem Wert.

Nicht unerwähnt bleiben sollen auch die Kompositionslehren von Printz (1696) Walther (1708), Kirnberger (1776-79) und Koch (1782-93). Für Modellsucher vielleicht interessanter noch sind drei stilistisch retrospektive Improvisationsschulen des späten 18. bzw. frühen 19. Jahrhunderts:

- Johann Michael Wiedeburg, Der sich selbst informirende Clavierspieler (Halle 1765-67): eine Clavierschule für >Liebhaberı, die ihre Anleihen bei Carl Philipp Emanuel Bach mit Zinsen zurückzahlt. In zwei dicken Bänden hat Wiedeburg, der als Organist in der ostfriesischen Ortschaft Norden weitab vom musikalischen Weltgeschehen wirkte, vieles aufgeschrieben, was andere wohl nur ihren Privatschülern am Instrument verraten haben. Sollte es jemals möglich gewesen sein, Generalbass und `Fantasieren` aus einem Lehrbuch zu erlernen, dann mit Wiedeburg auf dem Notenpult!

- Johann Gottfried Vierling, Versuch einer Anleitung zum Präludieren für Ungeübtere (Leipzig 1794): im Gegensatz zu Wiedeburgs monumentalem Opus ein Improvisationslehrgang en miniature, der es auf 29 Seiten von der Oktavregel über die Auflösung verschiedener `Gänge` des Generalbasssatzes in »kürzere Noten« bis zum galanten `Vorspiek schafft.

- Johann Christian Kittel, Der angehende praktische Organist (Erfurt 1803-08): Natürlich lassen sich auch aus den Kompositionslehren Kirnbergers und Kochs Modelle für

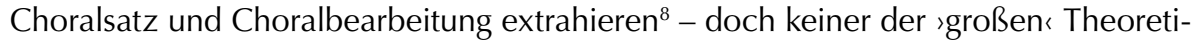
ker bietet dem sangehenden Organisten s eine so praxisnahe, auf ein überschaubares Modellrepertoire gestützte Lehre der Choralimprovisation wie Kittel.

7 Vgl. Riepel 1752-86, Kap. 4, »Erläuterung der betrüglichen Tonordnung«.

8 Vgl. Kirnberger 1776-79, insbes. Bd. 2, 1. Abschnitt, 3-40 sowie Koch 1782-93, Bd. 1, 257-366. 
Wie viel Mühe und Kosten hätten die heute verfügbaren Internetressourcen theoriegeschichtlich Interessierten meiner Generation ersparen können! ${ }^{9}$ (Andererseits: Bleibt nicht die Aura eines echten Buches unersetzlich?)

- Eine wahre Fundgrube ist die Digitale Bibliothek ${ }^{10}$ der Bayerischen Staatsbibliothek, die unter anderem Digitalisierungen einschlägiger Schriften von Printz, Murschhauser, Spiess, Sorge und Albrechtsberger zugänglich macht.

- Eine noch weit umfangreichere digitale Sammlung mit Schwerpunkten in der französischen und italienischen Musiktheorie stellt die Französische Nationalbibliothek ${ }^{11}$ zum Download bereit, darunter zentrale Schriften von Aaron, Gaffurio, Vicentino, Zarlino, Tigrini, Picerli und Rameau; auch Eitners nach wie vor relevantes Quellenlexikon ist verfügbar.

- Eine große Zahl italienischer Quellen des 16. und 17. Jahrhunderts in einem digitalen Textformat bietet der Thesaurus musicarum italicarum ${ }^{12}$, darunter Zarlinos Istitutioni harmoniche in der Ausgabe von 1589.

- Wichtige Generalbassquellen, darunter die Traktate von Banchieri, Penna, Muffat und Gasparini finden sich auf der Seite bassus-generalis.org ${ }^{13}$ des Genfer Konservatoriums.

- Verfügbar sind weiterhin Internet-Ausgaben von Berardis Documenti harmonici ${ }^{14}$ sowie des von Purcell überarbeiteten Kontrapunkttraktats von John Playford ${ }^{15}$.

- In der Digitalen Bibliothek sind rechtzeitig zum GMTH-Kongress in Freiburg 2007 Schriften von Riepel, Koch, Daube und Scheibe ${ }^{16}$ auf DVD erschienen. Meine Wunschtitel für etwaige Folgeausgaben: die bislang unveröffentlichten Schriften von Herbst und Vogt (gerne aber auch jene von Murschhauser und Spiess), Fenarolis Partimenti, Johann Gottfried Vierlings Anleitung zum Präludieren ${ }^{17}$ und die Wiener Generalbasslehren Voglers und Försters.

Als ich während meines Studiums Mitte der 90er Jahren die wissenschaftliche Literatur zur Theoriegeschichte sichtete, stieß ich fast ausschließlich auf musikwissenschaftliche Veröffentlichungen der 50er bis 80er Jahre - meist aus der Feder jener Autoren, deren Beiträge zu den Bänden der Geschichte der Musiktheorie bis heute Standards setzen. Viel

9 Alle hier angegeben Adressen waren am 6.11.2007 abrufbar.

10 http://mdz1.bib-bvb.de/ mdz/

11 http://gallica.bnf.fr/

12 http://euromusicology.cs.uu.nl/sitemap.html

13 http://www.bassus.cmusge.ch/

14 http://www.musica-antica.info/berardi/

15 http://ace.acadiau.ca/score/facsm/playford/

16 Kaiser 2007b.

17 Vierlings Anleitung zum Präludieren wurde nach dem Erscheinen dieser Kolumne neu ediert (Vierling 1794). 
hat sich seither nicht geändert: Die in den letzten zwei bis drei Jahrzehnten erfolgte Rezeption musikwissenschaftlicher Forschungsergebnisse durch die Musiktheorie darf nicht darüber hinwegtäuschen, dass in der `Nach-Dahlhaus-Äraı genuin theoriegeschichtliche Untersuchungen Mangelware geblieben sind. Das geradezu programmatische Desinteresse der jüngeren Musikwissenschaft an entsprechenden Fragen spiegelt sich in dem Diktum Karl Heinz Eggebrechts, die Quellen seien »in der Totalität ihrer Überlieferung bereits entdeckt und erschlossen « und aus guten Gründen erlahme jenes »traditionelle Geschichtsinteresse«, das »auf Kosten der Gegenwart" in die Vergangenheit zurückgetaucht sei. ${ }^{18}$ Doch kann bislang keine Rede davon sein, die Theoriegeschichte sei für die gegenwärtige Theoriebildung, geschweige denn für den Bereich der kunsthandwerklichpraktischen Instruktion, hinreichend aufgearbeitet: Der zeitgenössischen Musiktheorie böte sich die große Chance, mit selbstbewusstem Blick auf die eigene Fachgeschichte das Erbe der >old musicology zu integrieren.

Wissenschaftlich bislang noch wenig erschlossen ist beispielsweise die sneapolitanische Partimento-Tradition<, in der ein gesamteuropäisches, bis weit ins 19. Jahrhundert etabliertes Alternativmodell zur ramistischen Lehre wurzelte. Quellenlage, Traditionsund Wirkungsgeschichte harren einer umfassenden Aufarbeitung, und man darf die seit längerem angekündigten Beiträge von Ludwig Holtmeier und Giorgio Sanguinetti zum Thema mit Spannung erwarten.

- Eine grundlegende Einführung bietet Thomas Christensens Aufsatz »The ^Règle de I'Octave in Thorough-Bass Theory and Practice« (1992).

- Robert O. Gjerdingens jüngst erschienenes Lehrbuch Music in the Galant Style (2007) ist der erste Versuch, ausgehend von Modellen und Denkweisen der Partimentogestützten Ausbildungstradition eine sDogmatikı des galanten Stils zu schreiben. Im Zentrum steht die umfassend kommentierte und durch Analysen exemplifizierte Präsentation eines »Kernvokabulars« stilgebundener "Schemata«, die durchweg an das zeitgenössische bass- bzw. melodiestufenbezogene Tonalitätskonzept gebunden sind..$^{19}$ Die »kognitive Entwicklung « der musikalischen Elite des 18. Jahrhunderts, so Gjerdingen, sei geprägt durch das improvisatorische Training dieser Modelle und ihres kontextuellen Gebrauchs; zugleich sei die Gebundenheit an konventionelle Schemata Ausdruck einer »musikalischen Etikette» und somit Teil der zeitgenössischen, höfischen Musikästhetik. Der Anspruch, Konventionen einer Zeitsprache zu beschreiben, markiert zugleich die Stärken und Grenzen dieses imposanten Lehrbuchs: Man erfährt viel über das `was`, manches über das 'wie`, wenig über das 'Warum` und 'Woher`. Nicht ganz glücklich ist Gjerdingens Praxis, bislang namenlosen Modellen eigene, neue Bezeichnungen (etwa den Namen eines berühmten Lehrers oder Komponisten) zu verleihen: Neuprägungen wie »Prinner «, »Meyer « und »Fenaroli« stehen unvermittelt neben historischen Termini wie »Fonte« und »Monte«.

18 Eggebrecht 2000, 7.

19 Siehe auch:

http://faculty-web.at.northwestern.edu/music/gjerdingen/Papers/Presentations/Schemata.htm 
Eine Auswahl an Monuments of Partimenti (unter anderem von Leo, Durante und Fenaroli) hat Gjerdingen ins Netz gestellt. ${ }^{20}$ Im Druck sind bislang nur wenige Quellen zur Partimento-Tradition erschienen:

- Fedele Fenarolis bis ins späte 19. Jahrhundert unzählige Male wiederaufgelegte Partimenti, ossia basso numerato (Florenz 1863) sind leider nur bibliothekarisch zugänglich; die ursprünglich separat erschienenen Regole musicali di cembalo (Neapel 1775) entbehren der Notenbeispiele. Eine weitere zentrale Quelle, Francesco Durantes Bassi e Fughe ${ }^{21}$, liegt mittlerweile im Neudruck vor; mit Georg Muffats Regulae concentuum partiturae (1699) ist außerdem ein wichtiger Traktat aus dem Umfeld der frühen neapolitanischen Tradition greifbar.

- Johann David Heinichen, Der Generalbaß in der Composition (Dresden 1728): Heinichen gebührt das Verdienst, nicht allein die neue italienische Lehre für die Deutschen adaptiert, sondern auch als Erster den Zusammenhang von Bassstufe, Bassprogression und Bezifferung systematisch reflektiert und zu einem Teil der »musicalischen Wissenschaft " gemacht zu haben. ${ }^{22}$ Gewissermaßen als Kommentar zu Heinichen lässt sich Der Generalbaß-Satz und seine Rolle in Bachs Choral-Satz (1973) von Walter Heimann lesen: Mit Niedt und Heinichen werden die wichtigsten Verfechter einer Kompositionslehre auf Grundlage des Generalbasses gegen ihre Antipoden Mattheson (der dem Generalbass rein aufführungspraktische Relevanz zubilligte) und Kirnberger (den gelehrigen Schüler Rameaus) in Stellung gebracht.

- William Renwick, The Langloz Manuscript: Fugal Improvisation through Figured Bass (2001): ein wichtiges Zeugnis des mitteldeutschen Kontextes, dem Bach und Händel entstammen - nicht immer große Musik, aber eine überzeugende Antwort auf Renwicks Kernfrage: „Welche Art von Training befähigte einen Organisten des frühen 18. Jahrhunderts dazu, die Kunst der Begleitung und der Improvisation erfolgreich auszuüben? «²3

- David Ledbetter, Continuo Playing According to Handel: His Figured Bass Exercises $(1990)^{24}$ : kunstnahe Generalbassübungen mit einem klaren didaktischen Aufbau von elementaren Gängen bis hin zur Partimento-Fuge, niedergeschrieben von Händel für Prinzessin Anne (die älteste Tochter von Georg II.) - und von großem Wert für die gegenwärtige Unterrichtspraxis.

Die jüngere Fachliteratur hat Hans Aerts in seinem Beitrag zu »»Modell und `Topos« in der deutschsprachigen Musiktheorie« (2007) umfassend dargestellt. Einige Titel insbesondere auch aus dem anglo-amerikanischen Raum seien im Folgenden ergänzt:

20 http://faculty-web.at.northwestern.edu/music/gjerdingen/partimenti/index.htm

21 Durante 2003.

22 Heinichen 1728, insbes. 733-768 und 901-934.

23 Renwick 2001, Vorwort, ix.

24 Vgl. auch Mann 1964/65. 
- Daniel Harrison, »Rosalie, Aloysius and Arcangelo: A Geneaology of the Sequence» (2003): eine ausgezeichnete, systematische Darstellung des Modellrepertoires um 1700 mit Fokus auf Corelli.

- Heinrich Deppert, Kadenz und Clausel in der Musik von J. S. Bach (1993): Nirgendwo sonst wird der Zusammenhang von Klausellehre und Sequenztechnik so klar herausgearbeitet wie in Depperts penibel um historische Absicherung bemühtem »Exkurs über Sequenzen «. ${ }^{25}$

- Ulrich Kaiser, Die Notenbücher der Mozarts als Grundlage der Analyse von W. A. Mozarts Kompositionen 1761-1767 (2007a): Die Studie zu Satz- und Formmodellen in den Kompositionen des fünf- bis achtjährigen Mozart lässt sich als swissenschaftliches Pendant zu Gjerdingens Music in the Galant Style (2007) lesen (auch bei Gjerdingen findet sich ein Kapitel »The child Mozart«). Kaiser führt eindrucksvoll vor Augen, wie Mozarts Komponieren sich von Anbeginn im freien Spiel mit Konventionen entfaltete. Wertvoll sind seine "Methodologische[n] Vorüberlegungen", die den Begriff des Satzmodells (entgegen dem allgemeinen Gebrauch) eng an die Eigenschaft der »Formfunktion« (nach Caplin) in einem tonal-syntaktischen Kontext binden und damit von reinen Stimmführungsmustern abgrenzen.

Ob Schenkers Exegese von Carl Philipp Emanuel Bachs Ausführungen zur freien Fanta$\mathrm{sie}^{26}$, seine Aneignung der Fux'schen Theorie oder Riemanns teleologisch auf die Kategorien seines eigenen Denkens zielende Geschichte der Musiktheorie - stets wird es spannend, wenn die perspektivische Interpretation historischer Texte zugleich deren latente systematische Potentiale offenbart. Dies gilt auch für die gegenwärtige Beschäftigung mit Satzmodellen: Interessant sind Hartmut Fladts musikalische Topik ${ }^{27}$, Volkhardt Preuß' 'metahistorische` Anwendung der Klausellehre ${ }^{28}$ und Ludwig Holtmeiers Aufarbeitung der neapolitanischen Partimento-Tradition ja gerade, weil sie gegenwärtige Theorie im Dialog mit der Geschichte betreiben, also (mit den Worten Christensens) »die Theoriegeschichte« zur »historische[n] Basis gegenwärtigen Verstehens $\aleph^{29}$ machen. Manchmal gelingt ein solcher Dialog mit der Geschichte gewissermaßen unbeabsichtigt: Die Paradoxie, dass Schenker das salte`, melodisch-kontrapunktische Paradigma in einem dezidiert ahistorischen Strukturmodell gleichwohl kongenial fortgeschrieben hat, lässt Lehrbücher der Schenker-Nachfolge nicht selten in eine frappierende Nähe zu historischen Denkweisen und Darstellungsformen geraten:

25 Deppert 1993, 152-169.

26 Schenker, 1925, Bd. 1, 12-35.

27 Fladt 2005.

28 Preuß 1991.

29 »From this view, then, the history of theory takes on a critical new responsibility that far exceeds its charge as custodian of tradition; it defines the very historical basis upon which present understanding - contemporary music analysis - takes place.« (Christensen 1993, 32) 
- Felix Salzer und Carl Schachter, Counterpoint in Composition (1969): in mancher Hinsicht das englischsprachige Äquivalent zu Ulrich Kaisers modellgestützter Gehörbildung (die freilich ein völlig andersartiges didaktisches Konzept entfaltet): Was 1998 in Deutschland zu Recht Furore machte, ist in den USA seit 30 Jahren Standard.

- Allen Forte und Steven E. Gilbert, Introduction to Schenkerian Analysis (1982): als Analyselehrbuch nur eingeschränkt zu empfehlen, aber mit einem beachtenswerten Kapitel zu »Linear Intervallic Patterns «. ${ }^{30}$

- William Renwick, Analyzing Fugue: A Schenkerian Approach (1995): eine stimmige Synthese schenkerianischer Denkweisen mit einer konsequent historischen Perspektivierung. Modelle werden abhängig von ihrem formalen Kontext als »Exposition Patterns«, »Sequence Patterns" und »Stretto Patterns« behandelt. Die Kontextualisierung des als »Voice-leading Matrix« bezeichneten `Ursatzes« durch die barocke Klausellehre (nach Werckmeister) und die Lehre vom doppelten bzw. mehrfachen Kontrapunkt offenbart latente theoriegeschichtliche Bezüge.

Weitere Horizonte eröffnen sich, werden Fragestellungen und Methoden anderer Wissenschaftsbereiche, etwa der Kognitionspsychologie, auf den theorie- oder kompositionsgeschichtlichen Gegenstand bezogen:

- Roland Eberlein und Jobst P. Fricke, Kadenzwahrnehmung und Kadenzgeschichte - ein Beitrag zu einer Grammatik der Musik (1992): das Resumee der (im empirischen Teil nicht unangreifbaren) Studie: Eine »wahrnehmungsgerechte Analyse» tonaler Musik müsse sich auf stilübergreifende Stimmführungsmuster (insbesondere die Schemata der melodisch-kontrapunktischen Kadenzbildung) und die konkreten Gestalten des Intervallsatzes beziehen.

- Robert O. Gjerdingen, A Classic Turn of Phrase: Music and the Psychology of Convention (1988): ein großer interdisziplinärer Brückenschlag zwischen Kognitionspsychologie und Kompositionsgeschichte. Ausgehend von der >Lebensgeschichte emblematischen Melodieformel (8-7-4-3) zeigt Gjerdingen, wie überindividuelle musiksprachliche Schemata sich kognitiv verankern, wiedererkennbar und reproduzierbar werden.

Das Thema ‘Satzmodelle` ist ein Glücksfall für die Musiktheorie. Es zwingt geradezu, Geschichte (Theorie- und Kompositionsgeschichte), Systematik (Theorie) und Pragmatik (Improvisationslehre und historisch informierte Satzlehre) miteinander zu verknüpfen, und wäre überdies geeignet, der Musiktheorie einen Anschluss an entsprechende Fragestellungen der modernen Geistes- und Kulturwissenschaften zu vermitteln. Manche Anzeichen sprechen dafür, die deutschsprachige Musiktheorie sei auf dem Weg, einen echten Diskurs um Satzmodelle und Topoi zu eröffnen. 


\section{Literatur}

Aerts, Hans (2007), »'Modell und `Topos` in der deutschsprachigen Musiktheorie seit Riemann«, ZGMTH 4/1-2, 143-158.

Christensen, Thomas (1992), »The `Règle de l'Octave` in Thorough-Bass Theory and Practice«, Acta Musicologica 64, 91-117.

_ (1993), »Music Theory and its Histories«, in: Music Theory and the Exploration of the Past, hg. von David Bernstein u. Christopher Hatch, Chicago: University of Chicago Press, 9-39.

Deppert, Heinrich (1993), Kadenz und Clausel in der Musik von J. S. Bach. Studien zu Harmonie und Tonart, Tutzing: Hans Schneider.

Durante, Francesco (2003), Bassi e Fughe, Ms. Neapel, Neudruck, Padua: Armelin Musica.

Eberlein, Roland u. Fricke, Jobst P. (1992), Kadenzwahrnehmung und Kadenzgeschichte - ein Beitrag zu einer Grammatik der Musik (= Europäische Hochschulschriften, Reihe XXXVI, Bd. 79), Frankfurt a.M.: Peter Lang.

Eggebrecht, Hans Heinrich (2000), "Sinn von Musikwissenschaft heute«, AfMw 57, 3-8.

Fenaroli, Fedele (1775), Regole musicali di cembalo, Neapel, Reprint Bologna: Forni 1975 (= BMB II/140).

— (1863), Partimenti, ossia basso numerato, Florenz, Reprint Bologna: Forni 1967 (= BMB IV/61).

Fladt, Hartmut (2005), »Modell und Topos im musiktheoretischen Diskurs. Systematiken / Anregungen«, Musiktheorie 20, 343-369.

Forte, Allen u. Gilbert, Steven E. (1982), Introduction to Schenkerian Analysis, London/ New York: W.W. Norton \& Company.

Froebe, Folker (2007), „Satzmodelle des `Contrapunto alla mente` und ihre Bedeutung für den Stilwandel um 1600«, ZGMTH 4/1-2, 13-56.

— (2008), » Ein einfacher und geordneter Fortgang der Töne, dem verschiedene Fugen, Themen und Passagen zu entlocken sind . Der Begriff der sphantasia simplex bei Mauritius Vogt und seine Bedeutung für die Fugentechnik um 1700«, ZGMTH 5/2-3, 195-248.

Gjerdingen, Robert O. (1988), A Classic Turn of Phrase: Music and the Psychology of Convention, Philadelphia: University of Pennsylvania Press.

_ (2007), Music in the Galant Style, Oxford: Oxford University Press 2007.

Harrison, Daniel (2003), »Rosalie, Aloysius and Arcangelo: A Geneaology of the Sequence«, Journal of Music Theory 47, Yale University, 225-272.

Heimann, Walter (1973), Der Generalbaß-Satz und seine Rolle in Bachs Choral-Satz (= Freiburger Schriften zur Musikwissenschaft 5), München: Katzbichler.

Heinichen, Johann David (1728), Der Generalbaß in der Composition, Dresden, Reprint Hildesheim u. a.: Olms 1994.

Herbst, Johann Andreas (1643), Musica poetica, sive Compendium melopoëticum [...], Nürnberg, Mikrofiche, Leiden: IDC. 
Kaiser, Ulrich (1998), Gehörbildung. Satzlehre, Improvisation, Höranalyse. Mit einem Formkapitel von Hartmut Fladt (= Bärenreiter Studienbücher Musik 10-11), Kassel u. a.: Bärenreiter.

- (2007a), Die Notenbücher der Mozarts als Grundlage der Analyse von W. A. Mozarts Kompositionen 1761-1767, Kassel u. a.: Bärenreiter.

(2007b) (Hg.), Musiktheoretische Quellen 1750-1800, hg. von Ulrich Kaiser, Berlin: Zenodot Verlagsgesellschaft.

Kirnberger, Johann Philipp (1776-79), Die Kunst des reinen Satzes in der Musik, 4 Bde., Berlin und Königsberg, Reprint Hildesheim u.a.: Olms 1988.

Kittel, Johann Christian (1803-08), Der angehende praktische Organist, Erfurt, Reprint Leipzig: VEB Deutscher Verlag für Musik 1986.

Koch, Heinrich Christoph (1782-93), Versuch einer Anleitung zur Composition, 3 Bde., Rudolstadt und Leipzig, Reprint Hildesheim u. a.: Olms 1969.

Ledbetter, David (1990), Continuo Playing According to Handel: His Figured Bass Exercises, Oxford/New York: Oxford University Press (= Early music series 12).

Mann, Alfred (1964/65), »Eine Kompositionslehre von Händel«, Händel-Jahrbuch 10/11, Leipzig: VEB Deutscher Verlag für Musik, 35-57.

Marpurg, Friedrich Wilhelm (1753/54), Abhandlung von der Fuge [...], 2 Bde., Berlin, Reprint Hildesheim u. a.: Olms 1970.

(1755-60), Handbuch bey dem Generalbasse und der Composition, Berlin, Reprint Hildesheim u. a.: Olms 1974.

Muffat, Georg (1699), »Regulae concentuum partiturae«, Ms., hg. von Hellmut Federhofer als: An Essay on Thoroughbass (= Musicological Studies and Documents 4), American Institut of Musicology 1961. http://www.bassus.cmusge.ch

Murschhauser, Franz Xaver Anton (1721), Academia musico-poetica bipartita [...], Nürnberg, Mikrofiche, Leiden: IDC. http://mdz1.bib-bvb.de/ db/bsb00002470/images/

Preuß, Volkhardt (1991): Die Anwendung der Clausellehre des 17. Jahrhunderts im Theorieunterricht, Diplomarbeit, Hochschule für Musik und Theater Hamburg (unveröffentlicht).

Printz, Wolfgang Caspar: Phrynis Mitilenaeus oder Satyrischer Componist, 3 Bde., Dresden/Leipzig 1696, Mikrofiche, Leiden: IDC. http://mdz1.bib-bvb.de/ db/bsb 00002473/images/

Renwick, William (1995), Analyzing Fugue: A Schenkerian Approach, New York: Pendragon Press. (2001), The Langloz Manuscript: Fugal Improvisation through Figured Bass, Oxford/ New York: Oxford University Press.

Riepel, Joseph (1752-86), Anfangsgründe zur musicalischen Setzkunst, Regensburg und Wien, Reprint in: Joseph Riepel: Sämtliche Schriften zur Musiktheorie, Bd. 1, Wien u. a.: Böhlau 1996.

Salzer, Felix / Carl Schachter (1969), Counterpoint in Composition, New York, Reprint Chichester (West Sussex): Columbia Press 1989. 
Schenker, Heinrich (1925), Das Meisterwerk in der Musik, München: Drei Masken, Reprint Wien: Olms 1974.

Spiess, Meinrad (1745), Tractatus musicus compositorio-practicus, Mikrofiche, Leiden: IDC. http://mdz1.bib-bvb.de/ db/bsb00002469/images/

Sweelinck, Jan Pieterszoon (1901), Composition Regeln, Ms., hg. von Hermann Gehrmann, 's-Gravenhage/Leipzig (= Werken van Jan Pieterszn. Sweelinck 10).

Vierling, Johann Gottfried (1794), Versuch einer Anleitung zum Präludieren für Ungeübtere, Leipzig, Mikrofiche, Leiden: IDC, Neudruck ZGMTH 5/1-2, 373-392.

Vogt, Mauritius (1719), Conclave thesauri magnae artis musicae [...], Prag, Mikrofiche, Rochester, NY: Sibley Music Library.

Walther, Johann Gottfried (1708). Praecepta der Musicalischen Composition, Weimar, Neudruck hg. von Peter Benary, Leipzig 1955 (= Jenaer Beiträge zur Musikforschung 2).

Werckmeister, Andreas (1702), Harmonologia musica, Frankfurt und Leipzig, Reprint Hildesheim u.a.: Olms 1994.

Wiedeburg, Johann Michael (1765-67), Der sich selbst informirende Clavierspieler, 2 Bde., Halle, Reprint Wilhelmshaven: Noetzel 1996. 